\title{
Characteristics of Vertex Wave during Light Sleep according to Age
}

\author{
Sung Min Kim ${ }^{1}$, Gwan-Taek Lee ${ }^{1}$, Sun Jung Han $^{2}$ and Ki-Young Jung ${ }^{1}$ \\ ${ }^{1}$ Department of Neurology, Korea University Medical Center, Korea University College of Medicine, Seoul, Korea \\ ${ }^{2}$ Department of Neurology, Inam Neuroscience Research Center, Sanbon Medical Center, Wonkwang University College of Medicine, \\ Gunpo, Korea \\ 나이에 따른 얕은 수면에서의 정수리 파형의 특징 \\ 김성민 ${ }^{1}$, 이관택 ${ }^{1}$ 한선정 ${ }^{2}$, 정기영 ${ }^{1}$ \\ 고려대학교 의과대학 신경과학교실, ${ }^{1}$ 원광대학교 의과대학 산본병원 인암뇌신경연구센터 신경과학교실 ${ }^{2}$
}

Received December 18, 2011

Revised May 31, 2012

Accepted May 31, 2012

Address for correspondence

Ki-Young Jung, MD

Department of Neurology,

Korea University Medical Center,

Korea University

College of Medicine,

73 Inchon-ro, Seongbuk-gu,

Seoul 136-705, Korea

Tel: $+82-2-920-6649$

Fax: +82-2-925-2472

E-mail: jungky@korea.ac.kr
Objectives: Vertex wave during sleep is a hallmark of light sleep and related to brain development. We examined voltage characteristics of vertex wave according to age. Methods: Electroencephalographys were selected from routine waking and sleep EEG database. The inclusion criteria were that the age of subjects was more than 4 years old; EEG showed at least 5 typical vertex waves with no abnormal findings; the neurologic examination and neuroimaging findings were normal. EEGs were classified into 7 groups according to their age. Five to 20 epochs which included the time $320 \mathrm{~ms}$ before and after the maximum negativity of a vertex wave from each EEG record were selected. Voltage characteristics including amplitude and topographic distribution were evaluated. Intracerebral source location of vertex wave from each age group was identified using low resolution electromagnetic tomography (LORETA). Results: Amplitude of vertex wave was highest in the youngest age group, decreased with age thereafter. Voltage topography showed maximum negativity over central vertex area bilaterally in all age group, which did not show any significant difference among age group. Normalized amplitude of vertex waves showed significant main effect of location $(\mathrm{df}=1.356, \mathrm{~F}=$ 115.843, $\mathrm{p}<0.001)$. Interaction between age and location was also significant $(\mathrm{df}=4.088, \mathrm{~F}=3.327, \mathrm{p}=0.016)$. However, age did not show any main effect on amplitude of vertex wave. Post-hoc analysis showed amplitude of group 1 (5 to 9 years), compared with other age groups, was significantly lowered only in Fz electrode. LORETA image showed maximum current density in the mid to posterior cingulate gyri, which was similar across all age groups. Conclusions: Our study suggests that vertex waves might be related to arousal response during sleep, although exact mechanism is still unclear.

J Korean Sleep Res Soc 2012;9:5-9

\section{Introduction}

Vertex wave during sleep is usually diphasic, with an initial surface negative deflection followed by a low voltage surface positive phase. Vertex wave is bilaterally synchronous waves which have maximum negative amplitude at the vertex and often extend into frontal, temporal, and parietal areas. ${ }^{1}$

Vertex wave is a hallmark of sleep onset period. ${ }^{2}$ Gibbs and Gibbs were the first to emphasize age-related differences in sleep onset EEG in adults and children. They identified vertex waves (calling them biparietal humps) during sleep. Vertex wave appears in well developed form at the age of 5-6 months. ${ }^{3,4}$ It is reported that children are most likely to show parietal dominant vertex waves, while adult usually shows central maximum. However, only a few studies has been conducted to define amplitude and its topographic distribution according to brain development.

\section{Methods}

EEG was recorded from 19 scalp electrodes positioned according to the International 10-20 System referenced to $\mathrm{Pz}$ 
electrode. Then EEG signals obtained were re-referenced to a common average montage as described previously. ${ }^{5}$ Impedance was kept below $5 \mathrm{k} \Omega$, and the bandpass filter was set at $0.3-70 \mathrm{~Hz}$ with a sampling rate of $200 \mathrm{~Hz}$.

EEGs were selected from routine waking and sleep EEG database acquired from May to September, 2007 at Korea University Medical Center, Anam hospital. Inclusion criteria were followings; 1) age of subjects was from 4 to 70 years old; 2) EEG showed at least 5 typical vertex waves with no abnormal findings; 3) the neurologic examination and neuroimaging findings were normal. EEGs were classified into 4 age groups as followings; 5 to 9 years old (group 1), 10 to 29 years old (group 2), 30 to 49 years old (group 3), and 50 to 69 years old (group 4). Vertex waves were identified as bilaterally synchronous sharp transients in the central region during light sleep.

Five to 20 epochs which included the time $320 \mathrm{~ms}$ before and after the maximum negativity of a vertex wave at $\mathrm{Cz}$ electrode from each EEG record were selected. Voltage characteristics including amplitude and topographic distribution were evaluated. Baseline to peak amplitude was measured in common average montage in the present study.

Intracerebral source location of vertex wave from each age group was identified using the low resolution electromagnetic tomography (LORETA, The KEY Institute for Brain-Mind Research, University of Zurich, Switzerland). ${ }^{6}$ LORETA computes a unique three-dimensional electric source distribution by assuming that the smoothest of all possible inverse solutions is the most plausible, consistent with the assumption that neighbouring neurons are simultaneously and synchro-

Table 1. Number of subjects and of vertex waves at each age group

\begin{tabular}{lccccr}
\hline & $5-9$ year & $10-29$ year & $30-49$ year & $50-69$ year & Sum \\
\hline Subject (n) & 7 & 18 & 9 & 10 & 44 \\
Vertex (n) & 77 & 163 & 97 & 66 & 403 \\
\hline
\end{tabular}

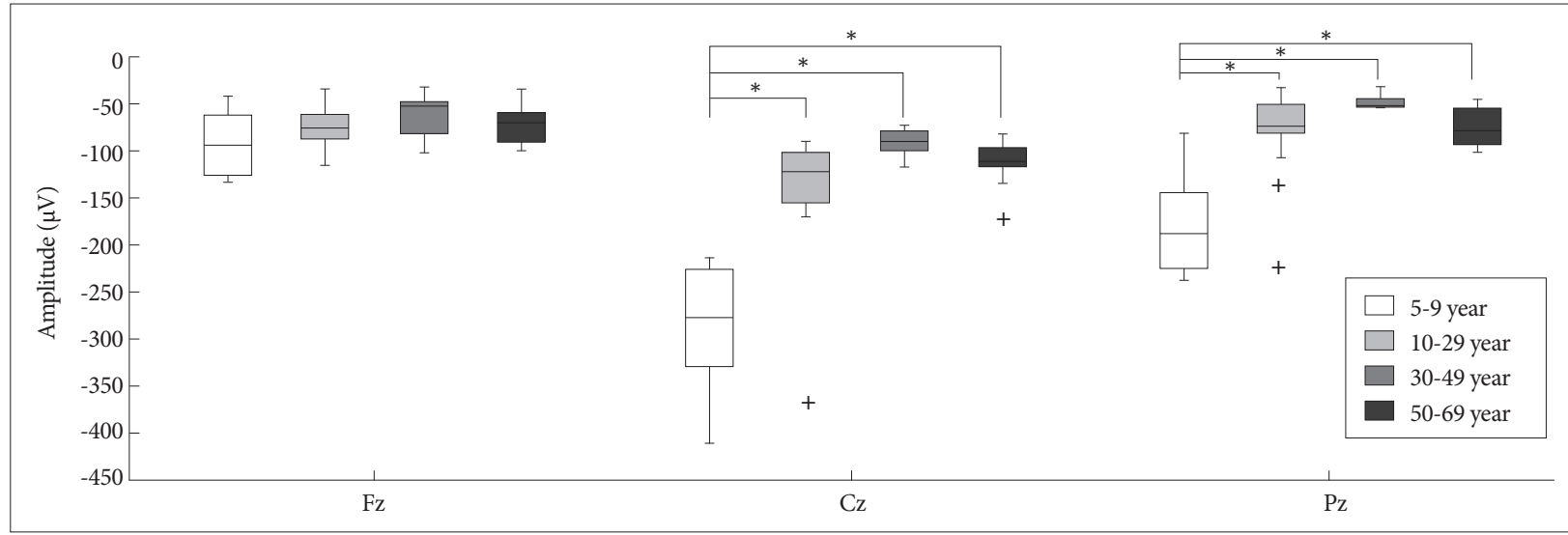

Fig. 1. Box-plot of amplitude of vertex waves within each group at the midline channels. Asterisks mean statistically significant results. The amplitude at $\mathrm{Cz}$ and $\mathrm{Pz}$ from youngest age group was significantly larger than those of the other age groups.

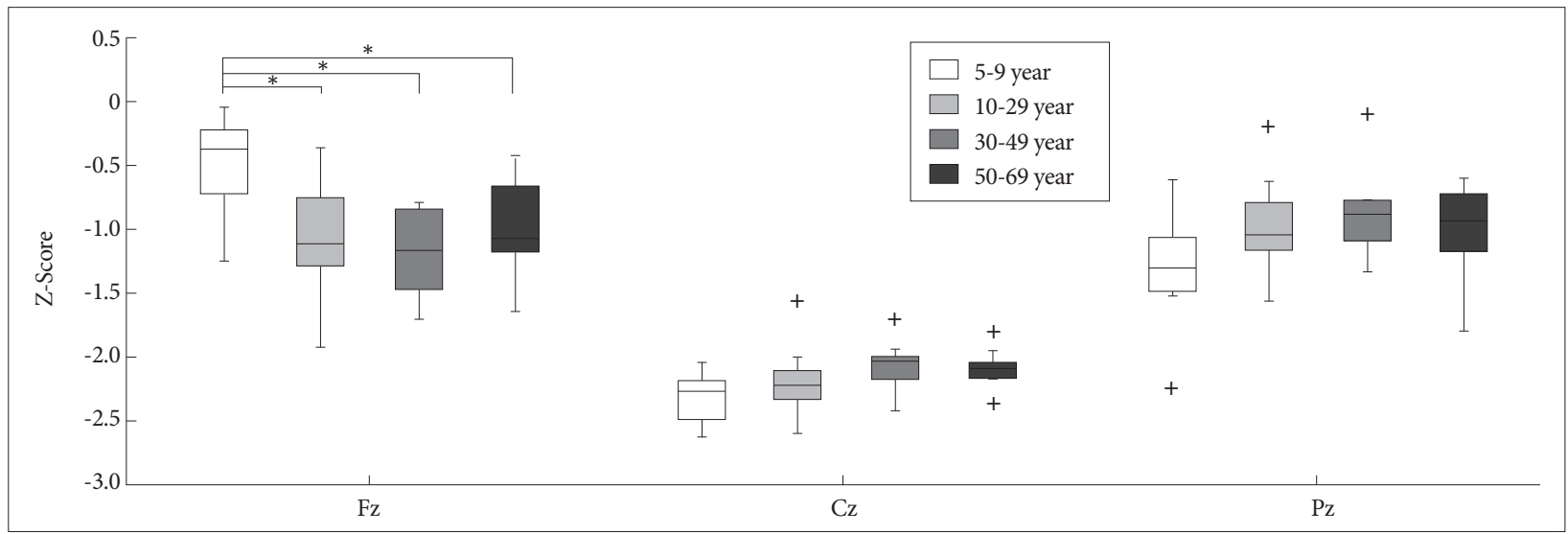

Fig. 2. Box-plot of normalized amplitude (z-score) of vertex waves within each group at the midline channels. The amplitude of youngest age group, compared with other age groups, was significantly lower only in Fz electrode. *significant level: $p<0.01$. 
nously active. LORETA images represent the current density in 2394 voxels with a spatial resolution of $7 \mathrm{~mm}$.

The amplitude and topographic distribution of the vertex wave was analyzed with repeated-measures analysis of variance (ANOVA). The within-subject factors was location (three levels: $\mathrm{Fz}, \mathrm{Cz}$ and $\mathrm{Pz}$ ) and between-subjects factor was age group (four levels: groups 1 to 4 ). The significant level of statistical tests was set to 0.05 . The Greenhouse-Geisser correction was used to evaluate $\mathrm{F}$ ratios to control Type 1 error in repeated measure design. Bonferroni post hoc tests were

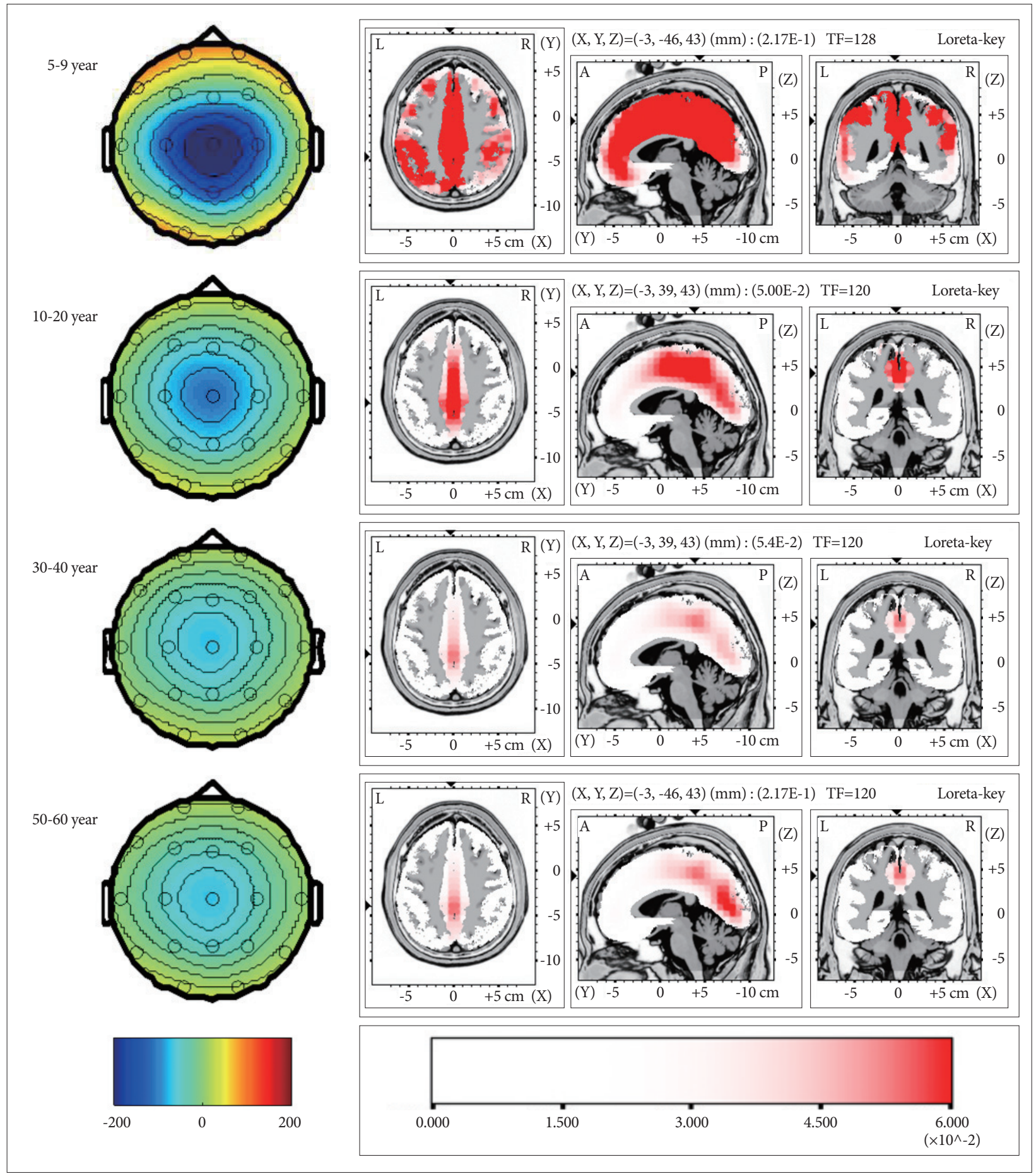

Fig. 3. Voltage topography (left panel) and low resolution electromagnetic tomography (LORETA) images (right panel) of vertex waves. Topographic distribution of vertex wave was quite similar pattern among all age groups. LORETA image shows maximum current density in the mid to posterior cingulate gyri, which was also similar across all age groups. 
used to identify the sources of significant repeated measures ANOVA.

\section{Results}

The number of subject and of vertex evaluated was presented in Table 1 . The amplitude of vertex wave was significantly different across locations ( $\mathrm{df}=1.072, \mathrm{~F}=73.176, \mathrm{p}<0.001)$. The amplitude of vertex wave was maximum over $\mathrm{Cz}$ electrode, followed by $\mathrm{Pz}$ in all age group (Fig. 1). Fz amplitude of vertex wave was the smallest in all age groups. Age showed significant main effect on the amplitude of vertex wave $(\mathrm{df}=3$, $\mathrm{F}=23.872, \mathrm{p}<0.001$ ). The interaction between age group and electrode location was also significant $(\mathrm{df}=5.106, \mathrm{~F}=11.598$, $\mathrm{p}<0.001$ ). The amplitude at $\mathrm{Cz}$ and $\mathrm{Pz}$ from youngest age group (i.e., group 1) was significantly larger than those of the other group. However, no significant difference was observed among group 2, 3, and 4. Fz amplitude was not different between age groups.

As amplitude of vertex wave has significant correlation with age (data not shown here), we calculated $z$-score to normalize any difference of voltage due to age. Normalized amplitude of vertex waves showed significant main effect of location $(\mathrm{df}=$ $1.356, \mathrm{~F}=115.843, \mathrm{p}<0.001)$. Interaction between age and location was also significant $(\mathrm{df}=4.088, \mathrm{~F}=3.327, \mathrm{p}=0.016)$. However, age did not show any main effect on amplitude of vertex wave. Post-hoc analysis showed amplitude of group 1 (5 to 9 years), compared with other age groups, was significantly lower only in Fz electrode (Fig. 2).

Although amplitude of vertex wave was different among age group, topographic distribution of vertex wave was quite similar among all age groups (Fig. 3). LORETA image showed maximum current density in the mid to posterior cingulate gyri, which was similar across all age groups.

\section{Discussion}

The aim of the present study was to identify amplitude and its topographic distribution of vertex waves according to subject's age. We found that amplitude of vertex wave was largest in subject whose age less than 10 years old in all midline electrodes, decreased with age thereafter. Voltage topography showed maximum negativity over central vertex area bilaterally in all age group, which did not show any significant difference among age group. However, one can argue that higher amplitude of vertex wave in younger age group may be due to thinner skull thickness. ${ }^{3}$ To avoid this problem, amplitude was normalized with $z$-score in the present study. Indeed, normalized amplitude of vertex wave at $\mathrm{Cz}$ and $\mathrm{Pz}$ was not different between age groups. However, normalized amplitude at $\mathrm{Fz}$ electrode showed a significant tendency to increase its amplitude from younger age group to middle-year age group.

It has been suggested that vertex waves constitute EEG response to afferent stimuli arising from interoceptors and exteroceptors. ${ }^{4}$ Either auditory stimuli or respiratory occlusion can evoke large negative component around $300-350 \mathrm{~ms}$ (N300 or N350) after stimulation during non-rapid eye movement sleep, which is identical to vertex waves in terms of waveform and topographic distribution. ${ }^{2,7}$ In light of this, vertex waves are considered as one of arousal response during sleep. ${ }^{8}$ It has been considered that arousal is a physiological phenomenon of cortical activation during sleep. In addition, it is reported that arousal index is linearly increased with age. ${ }^{9}$ In other words, arousal threshold of sleep is higher in young children than adult.

In our study, although normalized amplitude of vertex wave from $\mathrm{Cz}$ and $\mathrm{Pz}$ was no different between age groups, normalized $\mathrm{Fz}$ amplitude linearly increase with age from younger children to middle-aged subjects. It has been reported that frontal lobe is closely related to cortical arousal response. ${ }^{10}$ Therefore, lower amplitude of vertex wave of frontal region in children may suggest decreased response to arousal stimuli, hence decreased arousal response in children. To clarify the relationship between arousal response and vertex wave during light sleep, a larger study with polysomnographic data will be required to correlate vertex wave with variables pertaining to arousal responses such as arousal index, stage shifting, and cyclic alternating patterns.

In conclusion, our study suggests that vertex waves might be related to arousal response during sleep, although exact mechanism is still unclear.

\section{REFERENCES}

1. Fisch BJ, Spehlmann R. Fisch and Spehlmann's EEG Primer: Basic Principles of Digital and Analog EEG. 3rd ed. New York: Elsevier, 1999.

2. Bastien $\mathrm{CH}$, Crowley KE, Colrain IM. Evoked potential components unique to non-REM sleep: relationship to evoked K-complexes and vertex sharp waves. Int J Psychophysiol 2002;46:257-274.

3. Niedermeyer E, Silva FHL. Electroencephalography: Basic principles, clinical applications, and related fields. Philadelphia: Lippincott Williams \& Wilkins, 2005.

4. Ebersole JS, Pedley TA. Current Practice of Clinical Electroencephalography. Philadelphia: Lippincott Williams \& Wilkins, 2003.

5. Roh JH, Park MH, Ko D, et al. Region and frequency specific changes of spectral power in Alzheimer's disease and mild cognitive impairment. Clin Neurophysiol 2011;122:2169-2176.

6. Pascual-Marqui RD, Michel CM, Lehmann D. Low resolution electromagnetic tomography: a new method for localizing electrical activity in the brain. Int J Psychophysiol 1994;18:49-65.

7. Colrain IM, Webster KE, Hirst G, Campbell KB. The roles of vertex sharp waves and K-complexes in the generation of N300 in auditory and respiratory-related evoked potentials during early stage 2 NREM sleep. Sleep 2000;23:97-106.

8. Halász P, Kelemen A. Precipitatory role of arousal/antiarousal in nocturnal frontal lobe epilepsy. Clin Neurophysiol 2009;120:643; author re- 
Kim SM et al.

ply 643-644.

9. Boselli M, Parrino L, Smerieri A, Terzano MG. Effect of age on EEG arousals in normal sleep. Sleep 1998;21:351-357.
10. O'Malley EB, Norman RG, Farkas D, Rapoport DM, Walsleben JA. The addition of frontal EEG leads improves detection of cortical arousal following obstructive respiratory events. Sleep 2003;26:435-439. 